\title{
Measuring distances to Galactic SNRs using the red clump stars
}

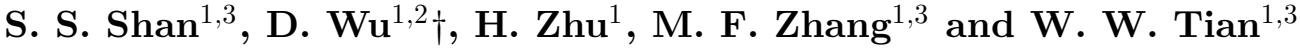 \\ ${ }^{1}$ National Astronomical Observatories, Chinese Academy of Sciences \\ Datun Road, Chaoyang District, Beijing 100012, China \\ email: tww@nao.cas.cn \\ ${ }^{2}$ College of Information Science and Technology, Beijing Normal University, \\ Beijing 100875, China \\ ${ }^{3}$ School of Astronomy, University of Chinese Academy of Sciences, \\ Beijing 100049, China
}

\begin{abstract}
Reliable distances to Galactic Supernova remnants (SNRs) are essential to constrain parameters that reveal the evolutional process of SNRs. We carry out a project to measure SNRs' distances in the first quadrant of the Galaxy. In this project, red clump stars (RCS) are used as standard candle to build the optical extinction $\left(A_{V}\right)-(\mathrm{D})$ distance relation in each direction of extinction-known SNRs. Here, G5.7-0.01, G54.1+0.3 and G78.2+2.1 are taken as typical examples. We obtain the distance of $3_{-0.3}^{+0.4} \mathrm{kpc}$ for G5.7-0.01, the lower limit of $5.8 \mathrm{kpc}$ for G54.1+0.3, the upper limit of $2 \mathrm{kpc}$ for G5.7-0.01. The results are consistent with distances from kinematic measurements. Hence, we highlight the RCS method can independently trace the distance to the SNRs.
\end{abstract}

Keywords. ISM: supernova remnants, methods: data analysis

\section{Introduction}

Distances of SNRs play important roles in obtaining basic parameters of SNRs like the age, the physical size, the expansion velocity and the explosion energy of the progenitor supernovae. There are several approaches to measure SNRs' distances such as kinematic method, proper motion measurement, Sedov estimate, and $\Sigma$-D relation. However, only about $20 \%$ of currently known Galactic SNRs have reliable distance measurements (Green 2014).

Red clump stars (RCS) are usually low mass stars in the early stage of core Heburning and concentrate in the color-magnitude diagram (CMD). The helium cores of RCS almost have the same mass. Meanwhile, the absolute magnitudes of RCS weakly depend on metal abundance in infrared band. Therefore, RCS are good enough to be standard candles. Durant \& van Kerkwijk (2006) measured the running of reddening with distance in the direction of Galactic anomalous X-ray pulsars and successfully obtained the distances of 5 pulsars. Güver et al. (2010) gave a distance estimate to the Low-mass $\mathrm{X}$-ray binaries $4 \mathrm{U}$ 1608-52 through the interstellar extinction traced by RCS. Zhu et al. (2015) used the similar measurement to determine the distance of SNR G332.5-5.6. We closely follow this method to systematically measure the distances to SNRs in the first Galactic quadrant. Here, we discuss RCS method and the results of SNRs G5.7-0.01, $\mathrm{G} 54.1+0.3$ and $\mathrm{G} 78.2+2.1$.

$\dagger$ S. S. Shan and D. Wu are common first author for the paper. 

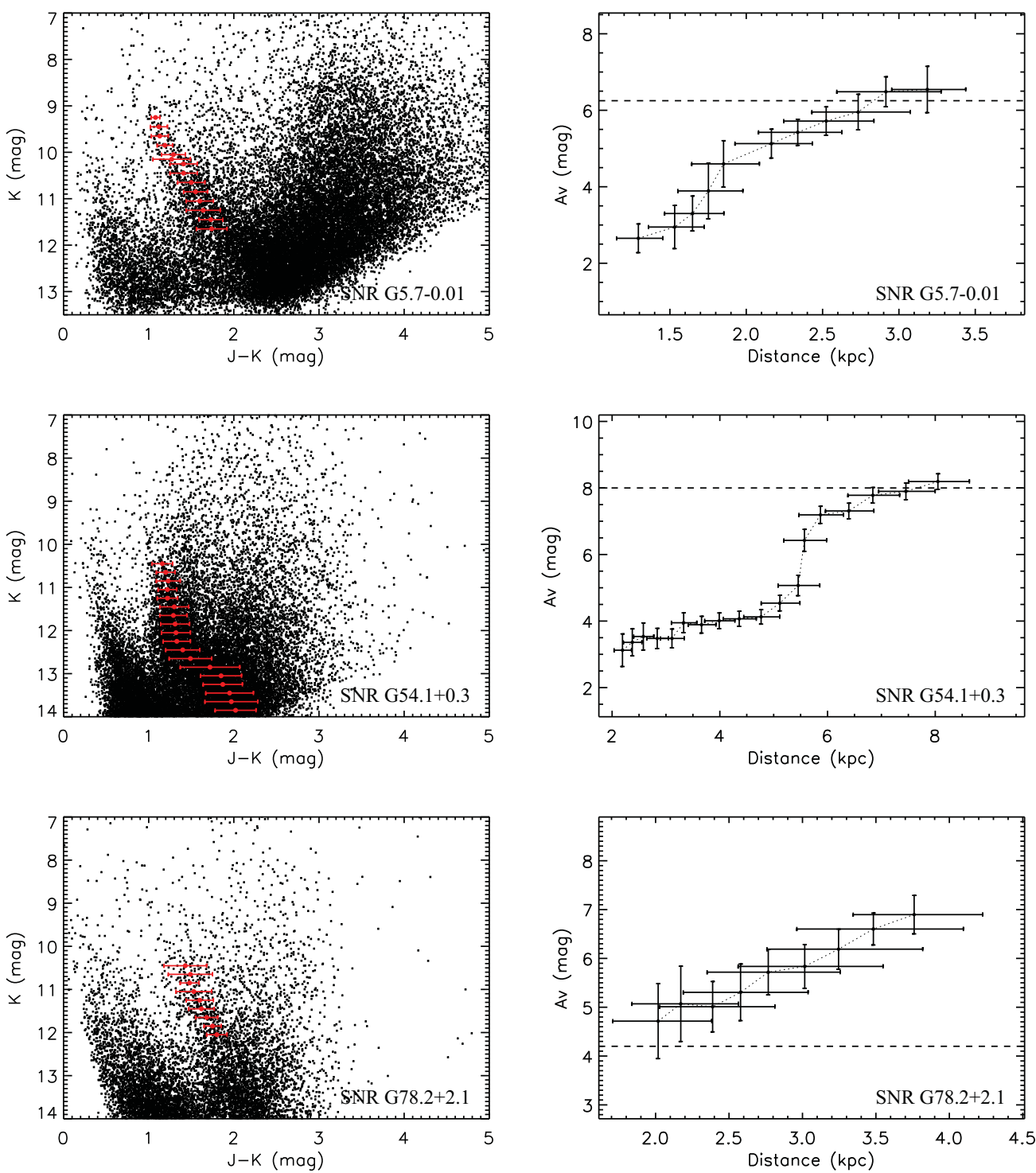

Figure 1. Left column: color-magnitude diagram for stars within $0.5 \mathrm{deg}^{2}$ of SNR G5.7-0.01, G54.1+0.3 and G78.2+2.1, respectively. The red dot and lines show the fitted location of the RCS density peak and its extent with $1 \sigma$. Right column: The derived $A_{V}$-D relation along the direction of G5.7-0.0, G54.1+0.3 and G78.2+2.1. The dashed line is $A_{v}$ value in the direction of an SNR.

\section{Method}

Firstly, we select the stars in the $0.5 \mathrm{deg}^{2}$ area around the center of a SNR in an interval of (Galactic longitude) $\triangle l 1^{\circ} \times \triangle b 0.5^{\circ}$ (Galactic latitude) from the 2MASS Allsky Point Source Catalog in the $J$ and $K_{s}$ (hereafter K) band (Skrutskie et al. 2006). Then, their magnitudes in $\mathrm{J}$ and $\mathrm{K}$ bands are used to construct the CMD (K vs. J-K). The RCS concentrate in a distinct region of the CMD (see Fig. 1, left column). The bulk of stars in the left region of the CMD are predominantly main sequence stars; while those in the right region are mainly dwarfs and RGBs. Secondly, we divide the stars in $m_{K}$ into successive strips. The width of each strip is usually 0.3 mag. It wil be extended 
Table 1. Distances measured by RCS method and parameters compiled from literatures.

\begin{tabular}{|c|c|c|c|c|c|c|c|}
\hline $\begin{array}{l}\text { Sourse } \\
\text { Name }\end{array}$ & $\begin{array}{c}\mathbf{N}_{H} \\
\left(\mathbf{1 0}^{21} \mathrm{Hcm}^{-2}\right)\end{array}$ & $\begin{array}{c}\mathbf{A}_{V} \\
(\mathbf{m a g})\end{array}$ & Model & $\begin{array}{c}\mathbf{D}_{k n \text { n } n} \\
\quad \mathbf{k p c}\end{array}$ & Method & Ref. & $\begin{array}{c}\mathbf{D}_{(t h i s w o r k)} \\
\mathbf{k p c}\end{array}$ \\
\hline G5.7-0.01 & $13 \pm 1$ & & Thermal plasma & 3.2 & kinematic measurement & $(1)(2)$ & $3_{-0.3}^{+0.4}$ \\
\hline G5 $54.1+0.3$ & & $8.0 \pm 0.7$ & Spectra fits & 6.2 & kinematic measurement & $(3)(4)$ & $>5.5$ \\
\hline $\mathrm{G} 78.2+2.1$ & & $4.2 \pm 0.8$ & $H_{\alpha} / H_{\beta}$ & $1.7-2.6$ & kinematic measurement & (5) (6) & $<2$ \\
\hline
\end{tabular}

Reference: (1) Joubert et al. (2016); (2) Hewitt \& Yusef-Zadeh (2009); (3) Koo et al. (2008); (4) Leahy et al.(2008); (5) Mavromatakis (2003); (6) Leahy et al. (2013)

to $0.5 \mathrm{mag}$ when the RCS are few. To locate the peak density of RCS in one strip, we adopt a Gaussian function to fit the RCS distribution and a power law function to fit the background stars. Then, the best fit produce color index $(\mathrm{J}-\mathrm{K})_{\text {peak }}$ at the peak density of the RCS distribution. Thirdly, we derive the average extinction for certain range of $\mathrm{K}$ by equation 2.1(Güver et al. 2010). Finally, distance for RCS of this strip can be derived by equation 2.2. Assuming that the intrinsic color $(\mathrm{J}-\mathrm{K})_{0}$ is $0.67 \mathrm{mag}$ and the mean absolute magnitude of RCS $\left(M_{K}\right)$ band is $-1.61 \mathrm{mag}$.

$$
\begin{aligned}
& A_{V}(\mathrm{mag})=\frac{A_{K}}{0.1137}=\frac{0.67\left[(J-K)_{\text {peak }}-(J-K)_{0}\right]}{0.1137} \\
& D(p c)=10^{\left[0.2\left(m_{K}-M_{K}+5-0.1137 A_{V}\right)\right]}
\end{aligned}
$$

This process is repeated for each stripe until reaching the 2MASS completeness limit. A series of extinction and distance of RCS are produced, then the $A_{V}-\mathrm{D}$ relation is built in the line-of-sight direction of an SNR (see Fig. 1, right column).

\section{Results and Discussion}

We build the $A_{V}$-D relation in the line-of-sight direction of SNRs G5.7-0.01, G54.1+0.3 and G78.2+2.1. In principle, an extinction value indicates one distance in a certain direction since the extinction increases with the growing distance. Overlapping the $\mathrm{A}_{V}$ values of SNRs with the corresponding $A_{V}$-D relation, distance are obtained. The parameters we used and the results are listed in Table 1.

For G5.7-0.01, its $A_{V}$ value is derived from the hydrogen column density $\left(N_{H}\right)$ since the theoretical and observational studies indicate that there is a relation between $A_{V}$ and $N_{H}$. We adopted the average value $A_{V} / N_{H}=(2.08 \pm 0.05) \times 10^{21} \mathrm{Hcm}^{-2} \mathrm{mag}^{-1}$ derived from 24 SNRs, 4 planetary nebulae and $65 \mathrm{X}$-ray binaries in the 1st and 4th Galactic quadrants, which is based on high quality data (Zhu et al. in prep.). In this case, the $A_{V}$ value in the direction of G5.7-0.01 is $6.25 \pm 0.48 \mathrm{mag}$, and the distance to G5.7-0.01 is estimated as $3.0 \mathrm{kpc}$.

According to the $\mathrm{A}_{V}-\mathrm{D}$ relation in the direction of $\mathrm{G} 54.1+0.3$, there is an extinction jump around $5.5 \mathrm{kpc}$. It may be caused by a huge molecular cloud or the increasing population of $\mathrm{K}$ dwarf which makes it difficult to identify RCS. In this situation, a conservative estimate should be made as $5.5 \mathrm{kpc}$ for the lower-limit distance.

On the CMD built in the direction of G78.2+2.1, the density of RCS is too small to be identified when the brighter magnitude is below 10 mag. The upper-limit distance of G78.2+2.1 can be estimated as $2 \mathrm{kpc}$. Leahy et al. (2013) determined the distance of G78.2+2.1 from $1.7 \mathrm{kpc}$ to $2.6 \mathrm{kpc}$. Combining the two results, the distance to G78.2+2.1 is range from $1.7 \mathrm{kpc}$ to $2 \mathrm{kpc}$.

In summary, our results show that the three distances estimated by RCS method are consistent with those measured by kinematic method. Extinction measurement based 
on RCS provides a reliable and independent clue to the SNRs' distance. In the future, we will apply this method to systematically measure the distances to Galactic SNRs. With the SNRs' distances, we will further re-calibrate the $\Sigma$-D relation and calculate distance-dependent parameters of SNRs.

\section{Acknowledgements}

We all acknowledge supports from NSFC program (11473038, 11603039).

\section{References}

Durant, M. \& van Kerkwijk, M. H. 2006, ApJ, 1070, 1081

Green, D. A. 2014, Supernova Environmental Impacts, 188, 196

Güver, T., et al. 2010, ApJ, 964, 973

Hewitt, J. W. \& Yusef-Zadeh, F. 2009, ApJ, 694, L16

Joubert, T., Castro, D., Slane, P., \& Gelfand, J. 2016, ApJ, 816, 63

Koo, B.-C., et al. 2008, ApJ, 673, L147

Leahy, D. A., Tian, W. W., \& Wang, Q. D. 2008, AJ, 136, 1477

Leahy, D. A., Green, K., \& Ranasinghe, S. 2013, MNRAS, 436, 968

Mavromatakis, F. 2003, A\& A, 398, 153

Skrutskie, M. F., et al. 2006, AJ, 1163, 1183

Zhu, H., Tian, W. W., \& Wu, D. 2015, MNRAS, 3470, 3474

Zhu, H., Tian, W. W., Li, A., et al. 2017, submitted to MNRAS 